\title{
Curves of Small Genus on Certain K3 Surfaces
}

\author{
BY SIR PETER SWINNERTON-DYER
}

e-mail: hpfs100@dpmms. cam. ac .uk

(Received 04 February 2014; revised 06 January 2014)

Recent workers $[\mathbf{1}, \mathbf{3}]$ have proved density theorems about the rational points on K3 surfaces of the form

$$
V: X_{0}^{4}+c X_{1}^{4}=X_{2}^{4}+c X_{3}^{4}
$$

for certain non-zero values of $c$. Their arguments depend on the presence of at least two pencils of curves of genus 1 on $V$. Unfortunately the values of $c$ for which the argument works are constrained by the need to exhibit explicitly a rational point on $V$ which satisfies certain extra conditions; these in particular require it to lie outside the four obvious rational lines on $V$. It is therefore natural to ask whether there are other curves of genus 0 or 1 defined over $\mathbf{Q}$ on $V$. In the case $c=1$ there are known to be infinitely many such curves (see [2]), and for general rational $c$ the quadratic form $Q$ on the Néron-Severi group whose value is the self-intersection number takes the values 0 and -2 infinitely often. Naively one might expect the case $c=1$ to be typical; but this is not so. The main object of this paper is to prove the following result.

THEOREM 1. If $c$ is not in $\pm \mathbf{Q}^{* 2}$ the only absolutely irreducible nonsingular curves of genus 0 on $V$ defined over $\mathbf{Q}$ are the four obvious straight lines. The only such curves of genus 1 have degree 3, 4 or 6.

Once this has been proved, it is straightforward to deduce the corresponding result for curves of higher genus.

THEOREM 2. Suppose that $c$ is not in $\pm \mathbf{Q}^{* 2}$. For any fixed $g$ there are only finitely many classes in the Néron-Severi group of $V$ which contain absolutely irreducible nonsingular curves of genus $g$ defined over $\mathbf{Q}$.

The proof constructs a bound for the degree of such a class in terms of $g$. I believe that this finiteness property is essentially a number-theoretic phenomenon, and that nothing analogous happens over $\mathbf{C}$.

The first step in proving these theorems is to find the Néron-Severi group $\operatorname{NS}(\mathbf{Q})$ of $V$. It is well known that $\mathrm{NS}(\mathbf{C}) \otimes \mathbf{Q}$ for $V$ is spanned by the classes of the 48 lines - as is most simply deduced from the fact that the corresponding quadratic form $Q$ has rank 20. (In fact these classes span $\mathrm{NS}(\mathbf{C})$, but this is harder to prove.) It follows that $\mathrm{NS}(\mathbf{Q}) \otimes \mathbf{Q}$ is spanned by the classes of the following divisors, where $\sum$ denotes the sum of the conjugates over $\mathbf{Q}$.

$$
\begin{aligned}
& \Lambda_{0} \text { is }\left\{X_{0}=X_{2}, X_{1}=X_{3}\right\}, \quad \Lambda_{1} \text { is }\left\{X_{0}=-X_{2}, X_{1}=X_{3}\right\}, \\
& \Lambda_{2} \text { is }\left\{X_{0}=X_{2}, X_{1}=-X_{3}\right\}, \quad \Lambda_{3} \text { is }\left\{X_{0}=-X_{2}, X_{1}=-X_{3}\right\} ; \\
& \Gamma_{6} \text { is } \sum\left\{X_{0}=X_{2}, X_{1}=i X_{3}\right\}, \quad \Gamma_{7} \text { is } \sum\left\{X_{0}=-X_{2}, X_{1}=i X_{3}\right\}, \\
& \Gamma_{8} \text { is } \sum\left\{X_{0}=i X_{2}, X_{1}=X_{3}\right\}, \quad \Gamma_{9} \text { is } \sum\left\{X_{0}=i X_{2}, X_{1}=-X_{3}\right\}, \\
& \Gamma_{10} \text { is } \sum\left\{X_{0}=i X_{2}, X_{1}=i X_{3}\right\}, \quad \Gamma_{11} \text { is } \sum\left\{X_{0}=i X_{2}, X_{1}=-i X_{3}\right\} ;
\end{aligned}
$$


$\Gamma_{0}$ is $\sum\left\{X_{0}=r X_{1}, X_{2}=r X_{3}\right\}, \quad \Gamma_{1}$ is $\sum\left\{X_{0}=r X_{1}, X_{2}=-r X_{3}\right\}$,

$\Gamma_{4}$ is $\sum\left\{X_{0}=r X_{1}, X_{2}=i r X_{3}\right\}$ where $r^{4}=-c$;

$\Gamma_{2}$ is $\sum\left\{X_{0}=s X_{3}, X_{2}=s X_{1}\right\}, \quad \Gamma_{3}$ is $\sum\left\{X_{0}=s X_{3}, X_{2}=-s X_{1}\right\}$,

$\Gamma_{5}$ is $\sum\left\{X_{0}=s X_{3}, X_{2}=i s X_{1}\right\}$ where $s^{4}=c$.

We use small greek letters for the corresponding divisor classes, and we denote by $\pi$ the class of a plane section.

LEMMA 1. The classes $\lambda_{0}, \lambda_{1}, \lambda_{2}, \lambda_{3}, \pi, \gamma_{0}$ form a base for $\operatorname{NS}(\mathbf{Q})$.

Proof. For any class $\delta$ the self-intersection number $(\delta \cdot \delta)=2 p_{a}(\delta)-2$ is even; moreover, by Riemann-Roch a class with $p_{a}(\delta) \geqslant 0$ and $d=\operatorname{deg}(\delta)>0$ is effective. For certain divisor classes $\delta$ we shall use the notation

$$
\delta=a_{0} \lambda_{0}+a_{1} \lambda_{1}+a_{2} \lambda_{2}+a_{3} \lambda_{3}+a_{4} \pi+a_{5} \gamma_{0} .
$$

The $\gamma_{i}$ with $i>0$ all have this form. The corresponding values of the $a_{j}$, which can be deduced consecutively, are as follows; in each line the function in the last column can be used to derive the corresponding formula:

$$
\begin{array}{lrrrrrrll} 
& a_{0} & a_{1} & a_{2} & a_{3} & a_{4} & a_{5} & & \\
\gamma_{6} & -1 & 0 & -1 & 0 & 1 & 0 & \text { using } & X_{0}-X_{2} \\
\gamma_{7} & 0 & -1 & 0 & -1 & 1 & 0 & \text { using } & X_{0}+X_{2} \\
\gamma_{8} & -1 & -1 & 0 & 0 & 1 & 0 & \text { using } & X_{1}-X_{3} \\
\gamma_{9} & 0 & 0 & -1 & -1 & 1 & 0 & \text { using } & X_{1}+X_{3} \\
\gamma_{10} & -1 & 0 & 0 & -1 & 2 & -1 & \text { using } & X_{0} X_{3}-X_{1} X_{2} \\
\gamma_{11} & 2 & 1 & 1 & 2 & -2 & 1 & \text { using } & X_{0}^{2}+X_{2}^{2} \\
\gamma_{3} & 1 & -1 & -1 & 1 & 0 & 1 & \text { using } & X_{0} X_{1}+X_{2} X_{3} \\
\gamma_{1} & -2 & -2 & -2 & -2 & 4 & -1 & \text { using } & X_{0} X_{3}+X_{1} X_{2} \\
\gamma_{2} & -3 & -1 & -1 & -3 & 4 & -1 & \text { using } & X_{0} X_{1}-X_{2} X_{3} \\
\gamma_{4} & 2 & 2 & 2 & 2 & 0 & 0 & \text { using } & X_{0}^{4}+c X_{1}^{4} \\
\gamma_{5} & 2 & 2 & 2 & 2 & 0 & 0 & \text { using } & X_{0}^{4}-c X_{3}^{4}
\end{array}
$$

This table shows that $\mathrm{NS}(\mathbf{Q}) \otimes \mathbf{Q}$ is generated by the $\lambda_{i}, \pi$ and $\gamma_{0}$. The intersection-number matrix of these six classes is as follows:

$\begin{array}{rrrrrrr} & \lambda_{0} & \lambda_{1} & \lambda_{2} & \lambda_{3} & \pi & \gamma_{0} \\ \lambda_{0} & -2 & 1 & 1 & 0 & 1 & 4 \\ \lambda_{1} & 1 & -2 & 0 & 1 & 1 & 0 \\ \lambda_{2} & 1 & 0 & -2 & 1 & 1 & 0 \\ \lambda_{3} & 0 & 1 & 1 & -2 & 1 & 4 \\ \pi & 1 & 1 & 1 & 1 & 4 & 4 \\ \gamma_{0} & 4 & 0 & 0 & 4 & 4 & -8\end{array}$

which has determinant -256 . Hence if the theorem is false there must be a divisor class (not necessarily primitive) in $\mathrm{NS}(\mathbf{Q})$ which has the form (1) with the $a_{i}$ all half-integers but not all integers. If $a_{4}$ is not an integer, consideration of the $\left(\delta \cdot \lambda_{i}\right)$ shows that just one of $a_{0}$ and $a_{3}$, and just one of $a_{1}$ and $a_{2}$, is an integer; and then $(\delta \cdot \delta)$ is not an integer. Again, if $a_{4}$ is an integer then for the same reason both or neither of $a_{0}$ and $a_{3}$, and both or neither of $a_{1}$ and $a_{2}$, are integers; and if one pair are integers and the other pair are not, then $(\delta \cdot \delta)$ is an odd integer. But $(\delta \cdot \delta)$ must be an even integer. So there are only three cases to consider: the $a_{i}$ which are not integers must be 
(i) $a_{0}, a_{1}, a_{2}, a_{3}$ or

(ii) $a_{5}$ or

(iii) $a_{0}, a_{1}, a_{2}, a_{3}, a_{5}$.

If case (i) happened then $\delta=\left(\lambda_{0}+\lambda_{1}+\lambda_{2}+\lambda_{3}\right) / 2$ would represent a curve of degree 2 and genus 1; but no such curve can exist. Again, the only quadric which contains $\Lambda_{0}+\Lambda_{3}+\Gamma_{10}$ is $X_{0} X_{3}-X_{1} X_{2}=0$, and $\gamma_{0}=2 \pi-\lambda_{0}-\lambda_{3}-\gamma_{10}$. Hence $\Gamma_{0}$ is the only effective divisor in $\gamma_{0}$. But if case (ii) happened then $\delta=\gamma_{0} / 2$ would be effective; so case (ii) cannot happen. A similar argument using $\gamma_{3}=2 \pi-\lambda_{1}-\lambda_{2}-\gamma_{10}$ shows that (iii) is impossible; for the only quadric which contains $\lambda_{1}+\lambda_{2}+\gamma_{10}$ is $X_{0} X_{1}+X_{2} X_{3}=0$.

To prove Theorem 1 we need to introduce further effective divisors. To define a typical one of them, note that the plane

$$
X_{0}-X_{2}=r\left(X_{1}-X_{3}\right) \quad \text { where } \quad r^{4}=-c
$$

meets $V$ in the two lines $\Lambda_{0}$ and $\left\{X_{0}=r X_{1}, X_{2}=r X_{3}\right\}$ and the conic

$$
2 X_{2}^{2}+3 r X_{1} X_{2}-r X_{2} X_{3}+2 r^{2} X_{1}^{2}-r^{2} X_{1} X_{3}+r^{2} X_{3}^{2}=0,
$$

which is absolutely irreducible. We call the sum of this conic and its three conjugates $\Gamma_{12}$; its class is $\gamma_{12}=-4 \lambda_{0}+4 \pi-\gamma_{0}$ and its self-intersection number is -8 . Similar arguments show that the classes

$$
\begin{aligned}
& \gamma_{13}=-4 \lambda_{3}+4 \pi-\gamma_{0}, \\
& \gamma_{14}=-4 \lambda_{1}+4 \pi-\gamma_{1}=2 \lambda_{0}-2 \lambda_{1}+2 \lambda_{2}+2 \lambda_{3}+\gamma_{0}, \\
& \gamma_{15}=-4 \lambda_{2}+4 \pi-\gamma_{1}=2 \lambda_{0}+2 \lambda_{1}-2 \lambda_{2}+2 \lambda_{3}+\gamma_{0}, \\
& \gamma_{16}=-4 \lambda_{0}+4 \pi-\gamma_{2}=-\lambda_{0}+\lambda_{1}+\lambda_{2}+3 \lambda_{3}+\gamma_{0}, \\
& \gamma_{17}=-4 \lambda_{3}+4 \pi-\gamma_{2}=3 \lambda_{0}+\lambda_{1}+\lambda_{2}-\lambda_{3}+\gamma_{0}, \\
& \gamma_{18}=-4 \lambda_{1}+4 \pi-\gamma_{3}=-\lambda_{0}-3 \lambda_{1}+\lambda_{2}-\lambda_{3}+4 \pi-\gamma_{0}, \\
& \gamma_{19}=-4 \lambda_{2}+4 \pi-\gamma_{3}=-\lambda_{0}+\lambda_{1}-3 \lambda_{2}-\lambda_{3}+4 \pi-\gamma_{0}
\end{aligned}
$$

are all effective.

Suppose that $\Delta$, whose class $\delta$ is given by (1), is an absolutely irreducible curve on $V$ defined over $\mathbf{Q}$. Let $\mathbf{R}^{6}$ be the space whose coordinates are the $a_{i}$ and let $x$ denote distance on $\mathbf{R}^{6}$. Provided that $\Delta$ is not one of the lines $\Lambda_{i}$ we must have $(\delta \cdot \theta) \geqslant 0$ where $\theta$ is any one of the $\lambda_{i}$ or $\gamma_{j}$. In what follows we shall ignore the conditions coming from $\gamma_{4}$ and $\gamma_{5}$. Let $\mathcal{P} \subset \mathbf{R}^{6}$ be the closed set defined by the remaining conditions and let $\mathcal{P}_{0}=\mathcal{P} \cap\{d=1\}$. Since

$$
2 d=\delta \cdot\left(\lambda_{0}+\lambda_{1}+\lambda_{2}+\lambda_{3}+\gamma_{6}+\gamma_{7}\right)=\delta \cdot\left(\lambda_{0}+\lambda_{3}+\gamma_{0}+\gamma_{10}\right)
$$

the only point of $\mathcal{P} \cap\{d \leqslant 0\}$ is the origin; hence $\mathcal{P}$ is a cone with vertex at the origin, and $\mathcal{P}_{0}$ is a cross-section of it. The same relations show that $\mathcal{P}_{0}$ is bounded, so that it is a polytope. Since

$$
\left.\begin{array}{rl}
(\delta \cdot \delta)= & \frac{1}{4} d^{2}-\frac{1}{4}\left(a_{0}+a_{1}+a_{2}+a_{3}-4 a_{5}\right)^{2}-4 a_{5}^{2} \\
& -\left(a_{0}-a_{1}-a_{2}+a_{3}-2 a_{5}\right)^{2}-\left(a_{0}-a_{3}\right)^{2}-\left(a_{1}-a_{2}\right)^{2}
\end{array}\right\}
$$

is convex on $\mathcal{P}_{0}$, it attains its minimum only at vertices of $\mathcal{P}_{0}$. To find these, we can afford to proceed in a very vulgar way.

The faces of $\mathcal{P}_{0}$ are among the 22 hyperplanes $(\delta \cdot \theta)=0$, where $\theta$ is as above. Any vertex is the intersection of 5 or more of these hyperplanes; we therefore examine each of the 26334 sets of 5 hyperplanes. It turns out that for 1964 of these sets the hyperplane equations are 
linearly dependent; and of the remainder only 362 determine a point which lies in $\mathcal{P}_{0}$. There is some redundancy because some vertices lie on more than 5 faces, and we only obtain 82 distinct vertices. None of these have $(\delta \cdot \delta)<0$; and 14 have $(\delta \cdot \delta)=0$. After rescaling to make the $a_{i}$ integers with highest common factor 1 , the corresponding $\delta$ become

$$
\lambda_{0}+\lambda_{1}+\lambda_{2}+\lambda_{3}, \quad-\lambda_{0}-\lambda_{1}-\lambda_{2}-\lambda_{3}+2 \pi,
$$

four like $\pi-\lambda_{0}$ and two like each of

$$
\begin{aligned}
& 2 \lambda_{0}+\gamma_{0}, \quad \lambda_{0}-\lambda_{1}+\lambda_{2}+\lambda_{3}+\gamma_{0}, \quad-\lambda_{0}-\lambda_{1}-\lambda_{2}-3 \lambda_{3}+4 \pi-\gamma_{0}, \\
& -2 \lambda_{0}-2 \lambda_{2}-2 \lambda_{3}+4 \pi-\gamma_{0} .
\end{aligned}
$$

These last eight classes are mapped to each other by the obvious symmetries of $V$. This completes the proof of Theorem 1.

We now turn to Theorem 2. Let $A_{0}$ be a vertex of $\mathcal{P}_{0}$ at which $(\delta \cdot \delta)=0$, and let $c>0$ be an integer such that $A=c A_{0}$ has integer coordinates. Let $L$ be any line through $A_{0}$ which for small positive $x$ measured from $A_{0}$ lies in the closed polytope $\mathcal{P}_{0}$; and let $\epsilon>0$ be independent of $L$ and such that the part of $L$ with $0 \leqslant x \leqslant \epsilon \operatorname{lies}$ in $\mathcal{P}_{0}$. On $L$ we have

$$
f_{2}(L, x)=d^{2}(\delta \cdot \delta) / d x^{2}<0
$$

by (2); so $f_{1}(L)=d(\delta \cdot \delta) / d x>0$ on $L$ at $A_{0}$ because $(\delta \cdot \delta)>0$ for small positive $x$. By compactness there is a constant $C_{1}>0$ such that $f_{1}(L) \geqslant C_{1}$. Similarly there is a constant $C_{2}$ such that $f_{2}(L, x) \geqslant-C_{2}$ for $0 \leqslant x \leqslant \epsilon$. Hence

$$
(\delta \cdot \delta) \geqslant C_{1} x-\frac{1}{2} C_{2} x^{2} \geqslant \frac{1}{2} C_{1} x
$$

provided $0 \leqslant x \leqslant \epsilon_{1}=\min \left(\epsilon, C_{1} / C_{2}\right)$. Of course $C_{1}, C_{2}, \epsilon$ and $\epsilon_{1}$ may depend on the choice of $A_{0}$.

Now let $B$ be a point of $\mathbf{R}^{6}$ which corresponds to an absolutely irreducible curve of genus $g>0$ on $V$ defined over $\mathbf{Q}$, and let $B_{0}$ in $\mathcal{P}_{0}$ be the point on the line $O B$ at which $d=1$. Let $\mathcal{S}$ be the closed subset of $\mathcal{P}_{0}$ obtained by removing those points which are a distance less than $\epsilon_{1}$ from $A_{0}$ for some $A_{0}$. In $\mathcal{S}$ we have $(\delta \cdot \delta)>0$, so by compactness there is a constant $C_{3}>0$ such that $(\delta \cdot \delta) \geqslant C_{3}$ in $\mathcal{S}$. Hence if $B_{0}$ is in $\mathcal{S}$ then $d^{2} \leqslant(2 g-2) / C_{3}$. If $B_{0}$ is not in $\mathcal{S}$ then there is an $A_{0}$ such that $B_{0}$ is a distance $x_{0}$ from $A_{0}$ where $0<x_{0}<\epsilon_{1}$. The coordinates of $A_{0}$ have denominator at most $d$ and those of $B_{0}$ have denominator at most $c$; so at least one of the coordinates of $B_{0}$ differs from the corresponding coordinate of $A_{0}$ by $y$ where $(c d)^{-1} \leqslant|y| \leqslant x_{0}$. Hence $d x_{0} \geqslant c^{-1}$; and by (3)

$$
2 g-2=(\delta \cdot \delta) \geqslant \frac{1}{2} C_{1} x_{0} d^{2} \geqslant \frac{1}{2} C_{1} d c^{-1}
$$

at $B$. Hence in all cases $d$ is bounded when $g$ is fixed; and by (2) this gives bounds for all the $a_{i}$.

Acknowledgements. I am indebted to Ronald van Luijk for pointing out an error in an earlier draft.

\section{REFERENCES}

[1] R. PANNEKoEK. Topological aspects of rational points on K3 surfaces (Doctoral thesis, Leiden 2013).

[2] H.P.F. Swinnerton-DYeR. Applications of Algebraic Geometry to Number Theory. Proc. Sympos. Pure Math. XX (Amer. Math. Soc., 1971).

[3] P. Swinnerton-Dyer. Density of rational points on certain surfaces. Algebra Number Theory 7 (2013), 835-851. 